\title{
LA INVERSIÓN EN ENERGÍAS RENOVABLES EN ARGENTINA
}

Marina Recalde*

-1 1 papel de la energía, base biofísica del desarrollo, es analizado por diversos autores (p. ej., Reister, 1987; Tahvonen y Salo, 2001; Recalde y Ramos, 2012). Sin un adecuado abastecimiento no es posible alcanzar un alto grado de desarrollo económico y social, pero este se puede lograr de varias maneras y usando fuentes con distintos grados de impacto ambiental. Este aspecto ha cobrado relevancia en las últimas décadas con el reconocimiento, en el quinto informe del Panel Intergubernamental sobre Cambio Climatico (IPCC), del papel del sector en la emisión de gases de efecto invernadero (GEI), del calentamiento global (DOI/IEA, 2013) y sus orígenes antropogénicos y de su impacto futuro.

Debido a la importancia económica y ambiental del uso de las diversas fuentes, en su proceso de desarrollo los países procuran sustituir sus matrices energéticas para reducir la dependencia energética y el impacto ambiental. Así ocurrió en los países de la OCDE después de los años setenta cuando, debido a las crisis del petróleo, el precio de los recursos de los que dependían (en su mayoría importados) aumentó notablemente, lo que afectó su balanza energética y su economía. Además, en la mayoría de los países desarrollados, el compromiso para reducir la emisión de GEI y las iniciativas de mitigación han impulsado el uso de nuevas tecnologías que utilizan energías renovables menos contaminantes.

* Consejo Nacional de Investigaciones Científicas y Técnicas y Fundación Bariloche, Buenos Aires, Argentina, [mrecalde@fundacionbariloche.org.ar]. Las opiniones que aquí se expresan son responsabilidad de la autora y no necesariamente coinciden con las de la institución en la que trabaja. Fecha de recepción: 10-02-2015, fecha de revisión: 15-04- 2017, fecha de aceptación: 2704-2017. Sugerencia de citación: Recalde, M. “La inversión en energías renovables en Argentina", Revista de Economia Institucional 19, 36, 2017, pp. 231-254. DOI: https://doi.org/10.18601/01245996.v19n36.09. 
Por su parte, en los países en desarrollo el principal objetivo para sustituir la matriz energética era hasta hace poco asegurar el abastecimiento, reducir la dependencia energética y mejorar la red de electricidad en zonas alejadas y poco desarrolladas (Fouquet, 2013). Ese es el caso de Argentina, donde la sustitución se inició en los años cuarenta, durante el primer gobierno de Juan Domingo Perón, para sustituir carbón mineral importado y petróleo (en su mayoría importado) por gas natural y grandes centrales hidráulicas. Esa política buscaba reducir la dependencia y aumentar el autoabastecimiento, sin fuerte presencia de objetivos ambientales (Dubrovsky, 2004; Barrera, 2011). A finales de los ochenta se empiezan a ver indicios de promoción de fuentes renovables e incorporación del concepto de uso racional o eficiente, aunque aislados e intermitentes. $\mathrm{Y}$ se han desarrollado algunas iniciativas, desde mejorar la información mediante mediciones y estadísticas hasta adoptar regulaciones con instrumentos económicos para incentivar la inversión privada. Pero los instrumentos no han logrado un impacto significativo en la inversión en energías renovables para generar electricidad. Según la Compañía Administradora del Mercado Mayorista (cammesa), en 2013 el 63,8\% de la electricidad era generada por tecnologías térmicas con base en gas natural y derivados de petróleo, el $31 \%$ por fuerza hidráulica (grandes centrales y pequeños emprendimientos), el 4,4\% por energía nuclear y el 1,3\% por nuevas fuentes renovables (FNRE).

A pesar de que hoy se reconoce la importancia de promover las energías renovables y su potencial para mitigar el impacto ambiental, aún enfrentan el problema de mayores costos y menor rentabilidad relativa que las tecnologías convencionales maduras ${ }^{1}$, lo que ha dificultado su difusión en todo el mundo. Es en este marco que surge la opción de emplear instrumentos que aumenten o aseguren la rentabilidad futura de estas inversiones, los cuales hoy se utilizan en muchos países (Jacobs et al., 2013; del Río y Mir, 2014; del Río y Linares, 2014; REN21, 2015). No obstante, la revisión empírica muestra que, aun aplicando instrumentos similares, los países logran distintos grados de inversión en energías renovables. Cabe entonces preguntar cuáles son los factores que afectan la política energética y los proyectos de energías renovables. Se trata, en su mayoría, de factores políticos, económicos e institucionales que enmarcan las decisiones de política

\footnotetext{
${ }^{1}$ Existe un intenso debate sobre la rentabilidad relativa de las FNRE y las tecnologías que usan recursos fósiles: algunos autores argumentan que hay subsidios encubiertos a estos recursos y que no se tienen en cuenta, en forma expresa, las externalidades negativas de su utilización.
} 
e influyen decisivamente en su aplicación. Muchos de ellos apuntan al comportamiento de la inversión y sus determinantes.

El comportamiento de la inversión es uno de los factores que más inciden en la actividad económica de un país; por ello diversos estudios analizan qué determina en última instancia el deseo de invertir de los agentes económicos. Muchos de esos estudios encuentran que son las expectativas de los agentes acerca de la rentabilidad y la estabilidad futuras de sus inversiones, las cuales son afectadas directamente por la incertidumbre ${ }^{2}$ (Dixit y Pindyck, 1994; Kosacoff y Ramos, 2006; Parigi et al., 2007; Mohn y Misund, 2009; Recalde, 2011a). En el caso de la promoción de energías renovables, la influencia de las expectativas es fundamental, pues suelen ser proyectos de alta inversión que requieren horizontes de planeación de largo plazo, en los que la estabilidad es esencial.

En este marco, este artículo analiza los factores del contexto argentino que están detrás de los instrumentos de promoción de energías renovables y que han llevado a su bajo desempeño. Argumenta que, en los países en desarrollo, el entorno de la política energética pude tener un efecto negativo que contrarresta el diseño y la aplicación de la política y de sus instrumentos. Las conclusiones que extraen son válidas para otros sectores, de modo que el análisis no se limita a un caso aislado, pues examina las condiciones que afectan a toda la economía y que operan de manera particular en el sector energético. No obstante, cada política sectorial opera en un entorno, cuyas condiciones hacen posible lograr o no resultados positivos. E1 marco institucional general afecta en forma diferencial a cada uno de esos marcos y, con ello, el resultado de la política en cuestión. Este artículo no se propone, entonces, encontrar las características específicas de la promoción de energías renovables, sino examinar de qué manera el marco institucional del país puede haberse opuesto a los instrumentos de política, así como explicar el escaso desarrollo de las energías renovables.

El artículo consta de cuatro secciones. Primero revisa las principales condiciones del entorno de la política de energías renovables y examina el efecto de las instituciones y de su interrelación con el desarrollo socioeconómico. Después presenta el método para estudiar las condiciones del entorno, con base en la adaptación de esquemas ampliamente utilizados en la economía ambiental y la economía

\footnotetext{
${ }^{2}$ Aquella situación en la que el futuro no se puede proyectar a partir del comportamiento anterior. Como argumenta Davidson $(1988 ; 1991)$, la incertidumbre es la característica básica de un mundo no ergódico, el mundo real. En los sistemas no ergódicos no se puede extrapolar el pasado al futuro, de modo que los agentes no pueden tener una idea exacta de los eventos futuros.
} 
institucional. En la tercera sección se analiza el entorno de la política de energías renovables en Argentina y el impacto de los factores institucionales. Por último, se presentan algunas reflexiones finales.

\section{EL ENTORNO Y LA PROMOCIÓN DE ENERGÍAS RENOVABLES}

En el diseño de la política energética interviene un conjunto de factores y elementos que llevan a su éxito o su fracaso, entre ellos: barreras a la entrada de las tecnologías, actores clave, instrumentos (directos e indirectos), condiciones nacionales e internacionales (o del entorno) (OLADE/CEPAL/GTZ, 2003).

Las barreras a la entrada son los obstáculos que enfrenta la introducción y el desarrollo de nuevas técnicas, los cuales dependen del tipo de tecnología y del grado de desarrollo de los países (Painuly, 2001; Painuly y Fenhann, 2002; Altomonte, 2007). Esas barreras se pueden eliminar con instrumentos adecuados: desde aquellos que permiten el acceso de las fuentes renovables a la red hasta aquellos que aumentan la masa crítica de capital humano requerido para su desarrollo. No obstante, puesto que en los países en desarrollo las fuentes renovables no enfrentan una barrera única sino un conjunto de barreras, es necesario recurrir a un conjunto de medidas que se complementen cuidadosamente para que las lleven por un sendero de expansión y desarrollo en el mediano y el largo plazo (Foxon et al., 2007).

Aunque el diseño de la política considere esas barreras y diseñe instrumentos adecuados para eliminarlas, hay otros factores externos a la política energética que determinan el resultado: las condiciones del entorno (condiciones habilitantes o condiciones de marco), aquellas que enmarcan la política energética y que no se pueden modificar fácilmente con estrategias, programas o instrumentos (OLADE/CEPAL/ GTZ, 2003). Entre ellas, el marco institucional, regulatorio y político más amplio, que influye, determina o facilita la viabilidad de políticas sectoriales o subsectoriales (Boldt et al., 2012, 9-13). La existencia de condiciones propicias o habilitantes facilita la eliminación de las barreras al proporcionar un contexto propicio para las estrategias (ibíd., 9-12). En el sector energético, las condiciones del entorno cumplen un papel fundamental para alentar la inversión, en especial las condiciones institucionales y macroeconómicas. James (2011) muestra que la mayor parte de la financiación para invertir en energías renovables en América Latina se ha dirigido en los últimos años a países que los inversionistas consideran seguros por su institucionalidad y que han sido consistentes en sus políticas energéticas y económicas. Diversos estudios enfatizan que la existencia de condiciones propicias facilita 
el desarrollo de las energías renovables. En particular, la calidad institucional y el cumplimiento de las regulaciones, la capacidad de adaptación a nuevas tecnologías, el acceso a financiación, el conocimiento tecnológico y el capital humano ${ }^{3}$.

El cuadro 1 describe algunas de las principales condiciones del entorno que se enfrentan cuando se diseña la política de energías renovables.

\section{Cuadro 1}

Condiciones del entorno para el desarrollo de una política de FNRE

\begin{tabular}{ll}
\hline Categoría & \\
\hline & $\begin{array}{l}\text { Capacidad organizativa } \\
\text { institucional }\end{array}$ \\
& \\
Gobernanza & $\begin{array}{l}\text { Marco político, legal y } \\
\text { regulatorio }\end{array}$
\end{tabular}

Gobernanza regulatorio

Desarrollo de capacidades (I\&D)

Económicas

Condiciones

macroeconómicas

Acceso a financiación interna/externa y disponibilidad de fondos

Información para el diseño y la toma de decisiones

Información

Información para el monitoreo

Conciencia Reconocimiento de la pública relevancia de las FNRE
Descripción e impacto

Alto nivel de compromiso y liderazgo. FNRE prioritarias en la agenda energética.

Organización institucional fuerte y adecuada que estimule la inversión mediante políticas.

Sinergia con otras políticas sectoriales relacionadas con aspectos ambientales del sector energético.

Marcos regulatorios estables y claros, y cumplimiento efectivo de las normas y leyes.

Importancia de las normas sobre transferencia de tecnología.

Capacidades individuales e institucionales.

Sinergia entre capacidades individuales e institucionales. No solo se requieren capacidades individuales y de equipo, sino también mantenerlas en las instituciones.

La existencia de condiciones macroeconómicas propicias elimina obstáculos a la inversión. Estabilidad de las principales variables macroeconómicas: inflación, volatilidad o no de la tasa de cambio. Un clima de incertidumbre macroeconómica afecta el proceso de inversión.

El desarrollo e implementación de políticas de promoción requerirá de financiación pública y privada.

Disponibilidad y sistematización de suficiente información de calidad.

Información transparente y de calidad del sector energético y de la economía en general, la cual permite monitorear y evaluar las políticas, lo que hace posible ajustarlas en caso de que no obtengan los resultados deseados. Al mismo tiempo la existencia de información confiable proporciona incentivos a la inversión externa y de instituciones y organismos internacionales.

La mayor conciencia pública aumenta la demanda popular por la incorporación de energías renovables que son más sustentables, y apoya el diseño de políticas de desarrollo de estas fuentes.

Fuente: Bouille (1999), United Nations Environment Programme(2011) y Zaballa et al. (2014); elaboración propia.

3 Ver, p. ej., Cherni (2011, 113-128), van Dril y van Tilburg (2011, 226-233), Bloomberg New Energy Finance et al., (2014, 28-33, 50-58), Corrigan et al. (2014) y Recalde (2015, 1-15). 


\section{El entorno desde la perspectiva de la economía institucional}

La relación entre instituciones y desarrollo económico, que determina en gran medida las diferencias entre países y se manifiesta en su impacto en el desempeño económico y político, es ampliamente conocida (Acemoglu y Robinson, 2008). Se trata de una relación bidireccional, puesto que las instituciones establecen el desarrollo socioeconómico diferencial de los países, pero al mismo tiempo diferentes niveles de desarrollo económico se corresponden con diferencias institucionales así como con diferentes posibilidades de cambios institucionales (Yifu y Nunget, 1995). Como argumentan London y Santos (2007), los países pueden estar inmersos en círculos virtuosos de desarrollo (buena calidad institucional-alto nivel de desarrollo socioeconómico-mejoramiento institucional-aumento de la calidad del desarrollo socioeconómico) o viciosos (baja calidad institucional-subdesarrollo socioeconómicoprecarización institucional-refuerzo del subdesarrollo).

Cabe señalar que la definición de instituciones para este análisis va más allá de la síntesis del cuadro 1 , donde las condiciones institucionales solo se refieren a las características de las instituciones $\mathrm{u}$ organizaciones del sector energético. En el análisis institucional, las instituciones se definen como "las reglas, normas y estrategias conocidas por la sociedad que crean incentivos (positivos o negativos) para el comportamiento de los agentes en situaciones que se repiten periódicamente" (Crawford y Ostrom, 1995, en Polski y Ostrom, 1998, 3; Ostrom, 2010). Esas instituciones formales o informales determinan el comportamiento de los individuos o grupos en la sociedad, y constituyen el contexto nacional que enfrenta la política energética, así como la reacción de los agentes ante sus instrumentos. Es decir, el marco institucional de un país establece las bases en las que se asientan las condiciones del entorno de las FNRE.

Cabe distinguir dos grupos de instituciones relevantes para este estudio, las cuales están estrechamente relacionadas: las instituciones políticas, que definen la estructura de decisión y la agenda política, determinan las decisiones del Estado y definen y limitan las reglas; y las instituciones económicas, que definen los derechos de propiedad (London y Santos, 2007).

Los derechos de propiedad tienen un impacto directo sobre los incentivos para invertir. La inversión es el factor clave para la sostenibilidad del sistema económico en general y en particular para la del sector energético. Cuando planean invertir, los agentes deciden en función de los retornos esperados, en un futuro incierto. En palabras de Keynes, en un mundo incierto los “espíritus animales" de los agentes 
impulsan las decisiones de inversión. Si bien la incertidumbre es una característica normal de un mundo no ergódico como en el que vivimos, en el que los cambios estructurales hacen imposible extrapolar el pasado al futuro (Davidson, 1988; 1991), la incertidumbre se debe en parte a que los resultados de las acciones económicas dependen de la interacción entre agentes, y que estos actúan según sus expectativas sobre las acciones de los demás (Arestis, 1996; Carabelli, 1988). Más allá de reconocer la existencia de incertidumbre, su grado difiere entre sistemas económicos o países. Así, el grado de incertidumbre sobre el retorno futuro de la inversión depende también de las características de los países. Los de menor grado de desarrollo suelen tener mayores debilidades institucionales, son más proclives a crisis económicas y políticas, y más sensibles a las crisis internacionales, lo que aumenta la incertidumbre de su contexto económico (Recalde, 2011a). Mientras que en situaciones normales de incertidumbre las reglas de comportamiento (facilitadas por las instituciones) permiten predecir ciertas regularidades; en situaciones de crisis (económicas o políticas), en las que no funcionan las convenciones, aumenta la incertidumbre (Vatn, 2009). Según la definición anterior, unas instituciones bien definidas que funcionen apropiadamente crean incentivos positivos para que el comportamiento de los agentes se repita periódicamente, y así reducen la incertidumbre del contexto. En cambio, cuando es imposible seguir "convenciones o reglas" (muchas veces por fallas institucionales) que produzcan resultados más o menos predecibles, los agentes actúan según su propio espíritu, lo que tiene efectos significativos en el comportamiento de la inversión. Una apropiada definición de los derechos de propiedad no elimina, pero sí disminuye, el riesgo percibido por los agentes -en ambientes inciertos- en sus decisiones hacia el futuro y su capacidad para reaccionar. Es decir, la existencia (y el cumplimiento) de instituciones económicas crea un marco propicio para que los agentes sigan reglas fijas, lo que reduce la incertidumbre sobre el desempeño futuro.

Además, cuando los derechos de propiedad se definen correctamente y se garantiza el cumplimiento de los contratos, los costos de transacción disminuyen y aumentan los incentivos para que los agentes inviertan (London y Santos, 2007).

Aquí cabe señalar la importancia de que existan contratos de largo plazo que enmarquen las transacciones $y$, sobre todo, de su cumplimiento, cuya función para incentivar la inversión y contribuir al desempeño económico ha sido estudiada por varios autores (p. ej., North y Thomas, 1980, y Ostrom, 1996). Este aspecto es clave en los actuales sectores energéticos de casi todo el mundo (en particular, los 
de América Latina y Argentina), donde la mayoría de las inversiones es realizada por agentes privados, que toman sus decisiones teniendo en cuenta las oportunidades globales (Recalde, 2011a). A esto se suma que las inversiones en el sector energético suelen tener altos costos irrecuperables, de modo que la percepción de seguridad y estabilidad económica y política en el largo plazo es aún más fundamental.

Así, la calidad de las instituciones políticas y económicas determina las principales condiciones del entorno que alientan o frenan el desarrollo de las energías renovables en un país. Las condiciones asociadas a la gobernanza ${ }^{4}$ (en especial, la capacidad organizativa e institucional y el marco político, legal y regulatorio), las asociadas a los aspectos macroeconómicos y financieros, y las asociadas a la conciencia pública son determinadas en última instancia por la calidad institucional del país.

Con este marco se puede explicar la existencia o la falta de inversiones en energías renovables, así como el éxito o el fracaso de las políticas, con base en un conjunto de factores institucionales que determinan las condiciones del entorno para la política energética, por ejemplo, expectativas sobre el diseño institucional, existencia o ausencia de un horizonte temporal de largo plazo entre agentes políticos, garantía (o no) de los derechos de propiedad e (in)cumplimiento de los contratos.

Gráfica 1

Interacción entre inversión en FNRE y determinantes de la inversión

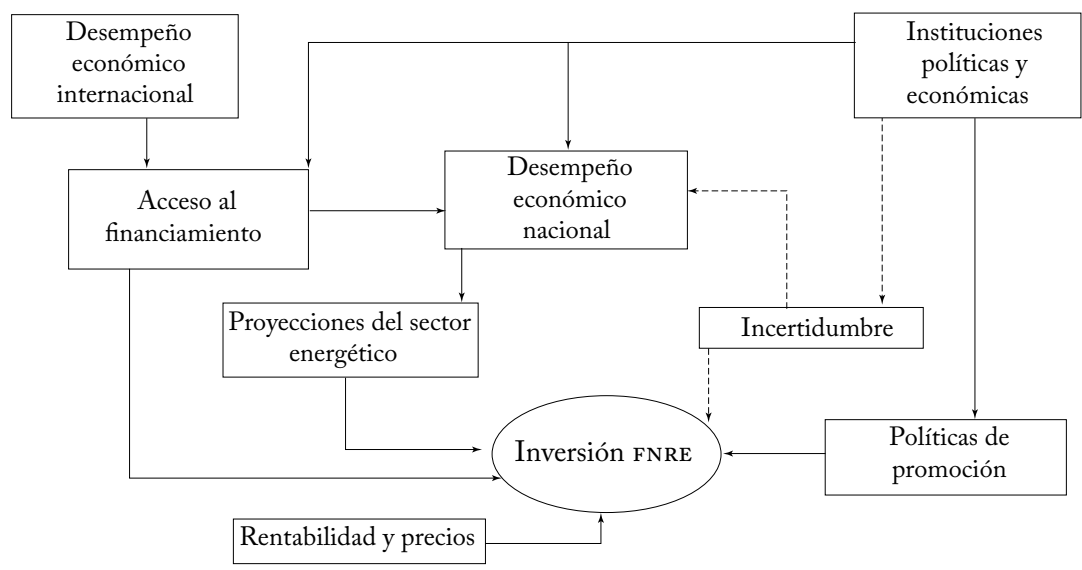

Fuente: adaptado de Recalde (2011a).

${ }^{4}$ Como señalan London y Rojas (2015), el concepto de "gobernanza" alude a un complejo proceso de toma de decisiones públicas que va más allá de la buena gestión de los recursos e incluye el diseño de un buen marco institucional. 
La gráfica 1 muestra un enfoque posible de los determinantes de la inversión en FNRE. Esta gráfica, una adaptación de los determinantes de la inversión en exploración de hidrocarburos, sintetiza la interrelación entre condiciones institucionales (políticas y económicas, tal como se definieron) y desarrollo de las energías renovables, y su impacto en la inversión (pública y privada). Tres relaciones merecen especial atención. Primera, el impacto positivo ya mencionado de la calidad institucional sobre el desempeño económico y el de un buen desempeño en el desarrollo de proyectos de FNRE. Este último se puede atribuir a un entorno propicio para la inversión y a que las proyecciones de crecimiento nacional involucran el crecimiento del sector energético (dada la alta elasticidad ingreso del consumo de energía, sobre todo en las etapas iniciales del desarrollo), el cual demanda inversiones en energía. Segunda, la relación indirecta entre calidad institucional e incertidumbre, y entre esta e inversiones en FNRE. En el marco que presentamos, cuanto mayor sea la calidad institucional, mayor será la posibilidad de que los agentes sigan reglas y convenciones, y menor será la incertidumbre que surge de la interacción entre sus expectativas. Entornos con menor grado de incertidumbre son más propicios para la inversión en todos los sectores, y aún más en aquellos que requieren largos horizonte de planeación y niveles de rentabilidad estables, como el sector energético y la inversión en FNRE. Tercera, el impacto de la calidad institucional en la ejecución de las políticas de promoción. Cuando mayor sea la calidad de las instituciones políticas, mayor será su capacidad para desarrollar políticas de promoción. Este aspecto es muy importante en el desarrollo de las FNRE porque la difusión de las energías renovables hoy depende en gran medida de los planes y programas de promoción, al menos en los países en desarrollo.

\section{MÉTODO PARA EVALUAR EL ENTORNO}

Como ya se mencionó, un marco propicio para el desarrollo de energías renovables incluye aspectos económicos, políticos y sociales. La comparación en el tiempo de estos marcos entre países y en un mismo país requiere un marco analítico que integre las distintas dimensiones. Un enfoque similar al que se requiere para evaluar situaciones en las que coexisten perspectivas socioeconómicas, ecológicas, institucionales y éticas, donde la medición tiene dificultades, como el análisis de las políticas ambientales (UNEP, 2011). Un enfoque de uso común en esas situaciones es el análisis multicriterio, AMC (DCLG, 2009), en el que se definen criterios atendiendo a los objetivos de política y se valora 
el alcance de cada criterio, así como su peso en un indicador global. Este tipo de análisis, que requiere la participación de los principales actores porque utiliza su información, es usual en la evaluación de tecnologías y en estudios del cambio climático.

En este trabajo se adoptó el AMc en su primera etapa para analizar las condiciones del entorno, pues no se realizó con participación de los agentes sino con información secundaria. Ante la falta de una valoración de los agentes clave, los criterios no se integraron en un indicador único, sino que se recurrió a una representación gráfica. Se presenta entonces un marco esquemático que permite ver cómo interactúan las condiciones del entorno para crear un entorno global que facilite (o no) la promoción de energías renovables con ayuda de un rodograma.

Para analizar las condiciones del entorno mencionadas en el cuadro 1 se tradujeron a indicadores, seleccionados por su capacidad para representar cada condición, por su simplicidad y por la disponibilidad de información. Siguiendo a Corrigan et al. (2014), las variables seleccionadas para cada indicador se agregaron y se pasaron a una escala de 1 a 5 , usando una transformación mín-máx para preservar la distancia entre puntajes.

La conversión de condiciones cualitativas en condiciones cuantitativas es uno de los aspectos más complejos del análisis. De modo que los indicadores seleccionados y su puntuación pueden ser modificados por otros autores, o en futuros trabajos para ajustarlos a cada estudio de caso. Cabe señalar, además, que para simplificar el análisis se les da igual peso a todos los indicadores (y, por ende, a todas las condiciones del entorno mencionadas). Esta es una debilidad metodológica porque no todas las condiciones tienen el mismo impacto, y se debe modificar en el futuro. En próximos trabajos se puede extender el análisis aplicando el MCA completo, usando información primaria recogida en encuestas o talleres donde los actores ayuden a definir los objetivos, la puntuación y la ponderación, y a elaborar así un índice de consenso.

En este escrito se usan principalmente datos cualitativos. Cada indicador se construyó con base en información secundaria: revisión y evaluación de la información energética disponible (balances energéticos, reservas, precios mayoristas, disponibilidad y potencial de FNRE, mapas solares y eólicos, otra información disponible, documentación y leyes sobre la promoción de energías renovables, metas de FNRE, instituciones para promoverlas, instrumentos, etc.).

\footnotetext{
${ }^{5}$ Estos esquemas son de uso común en la literatura energética y ambiental; ver CEPAL/OLADE/GTz (1997), Salgado y Altomonte (2001), IEE-USP-FB (2013), Rockström et al. (2009), Duguma et al. (2014) y Recalde (2015).
} 
Cuadro 2

Indicadores seleccionados. Definiciones y puntuación

\begin{tabular}{|c|c|c|}
\hline & Indicador & Definición y puntuación \\
\hline 1 & Compromiso & $\begin{array}{l}\text { ¿Cuál es el compromiso del gobierno con las FNRE? ¿Hay ex- } \\
\text { presiones oficiales de tal compromiso? Documentos o leyes que } \\
\text { establezcan la promoción de las FNRE como prioridad de política } \\
\text { nacional. ¿Ha emprendido el país alguna NAMA (National Appro- } \\
\text { piate Mitigation Action) o un proyecto de MDL (Mecanismo de } \\
\text { Desarrollo Limpio) relacionado con la promoción de las energías } \\
\text { renovables? ( } 1 \text { = muy baja; } 5 \text { muy alta) }\end{array}$ \\
\hline 2 & Instituciones & $\begin{array}{l}\text { ¿Qué tan desarrolladas están las instituciones de energías re- } \\
\text { novables? ¿Hay alguna especializada en la promoción de FNRE, } \\
\text { independiente o dependiente de algún organismo gubernamental? } \\
\text { ( } 1 \text { = muy poco desarrolladas, } 5=\text { muy desarrolladas y eficientes) }\end{array}$ \\
\hline 3 & Regulación & $\begin{array}{l}\text { ¿Existen políticas e instrumentos para promover fuentes de ener- } \\
\text { gía limpia? ¿Cuáles son las percepciones sobre la capacidad de } \\
\text { las instituciones gubernamentales para formular e implementar } \\
\text { políticas de energía limpia? } \\
\text { ( } 1 \text { = muy deficientes; } 5=\text { muy buenas) }\end{array}$ \\
\hline 4 & Sinergia & $\begin{array}{l}\text { ¿Cuenta el país con estrategias de desarrollo bajas en carbono } \\
\text { o de cambio climático que promuevan las FNRE? ¿Tiene el país } \\
\text { iniciativas de reducción de GEI o se encuentra planificándolas? } \\
\text { ( } 1 \text { = no hay; } 5 \text { = existen y el país ha emprendido MDL y NAMA) }\end{array}$ \\
\hline 5 & Economía & $\begin{array}{l}\text { Percepción de los inversionistas privados acerca de las condiciones } \\
\text { macroeconómicas. Cambios en los índices de precios al consumi- } \\
\text { dor, tasa de inflación anual. Deuda pública como porcentaje del pIB. } \\
(1=\text { condiciones macroeconómicas no favorables; } 5 \text { = muy favo- } \\
\text { rables) }\end{array}$ \\
\hline 6 & Financiación & $\begin{array}{l}\text { Derechos de propiedad y credibilidad nacional. ¿Cuán fuerte es la } \\
\text { protección de los derechos de propiedad, incluidas las inversiones } \\
\text { y los activos financieros? Probabilidad de entrar en situaciones } \\
\text { de default. } \\
\text { (1 = deficientes y alta probabilidad; } 5 \text { = buenos y baja probabilidad })\end{array}$ \\
\hline 7 & $\begin{array}{l}\text { Información } \\
\text { energética }\end{array}$ & $\begin{array}{l}\text { ¿Cuán buena y confiable es la información del sector energético? } \\
\text { Hay información suficiente y confiable de las principales variables } \\
\text { del sector al menos para } 10 \text { años (balances energéticos, reservas de } \\
\text { recursos energéticos, precios y capacidad instalada en los mercados } \\
\text { eléctricos mayoristas, etc.). } \\
(1=\text { no confiable; } 5 \text { = confiable y apropiada) }\end{array}$ \\
\hline 8 & $\begin{array}{l}\text { Información de } \\
\text { energías reno- } \\
\text { vables }\end{array}$ & $\begin{array}{l}\text { ¿Cuán buena y suficiente es la información sobre energías reno- } \\
\text { vables? Hay información gratuita disponible sobre dotación de } \\
\text { energías renovables: mapas eólicos y solares, información sobre } \\
\text { biomasa, bases de datos sobre costos de FNRE, etc. } \\
(1=\text { insuficiente; } 5=\text { suficiente y relevante })\end{array}$ \\
\hline 9 & Capacidades & $\begin{array}{l}\text { Hay programas públicos de educación, formación e investigación } \\
\text { para profesionales en el campo de las FNRE. } \\
(1=\text { insuficiente; } 5 \text { = suficiente })\end{array}$ \\
\hline 10 & $\begin{array}{l}\text { Conciencia } \\
\text { pública }\end{array}$ & $\begin{array}{l}\text { ¿Las instituciones gubernamentales hacen campañas de informa- } \\
\text { ción para promover el uso de FNRE en conjunto con otras políticas } \\
\text { de mitigación? } \\
(1=\text { muy pocas; } 5 \text { = muchas })\end{array}$ \\
\hline
\end{tabular}

Fuente: Recalde (2015), elaboración propia. 
Se recopiló información de la Secretaría de Energía y de la Secretaría de Medio Ambiente y Desarrollo Sustentable de la Nación. Para la información sobre las acciones de mitigación registradas se recurrió a la página oficial de la Convención Marco de las Naciones Unidas sobre Cambio Climático (cMNUCC). La información cuantitativa sobre aspectos macroeconómicos se obtuvo del Ministerio de Economía.

Esa información se complementó con la de cuatro estudios sobre la institucionalidad nacional: Climatescope 2014 (вNEF et al., 2014); el proyecto REN217; Global Competitiveness Index Report 2014-2015 (Corrigan et al., 2014) y World Governance Indicators (wgi) del Banco Mundial (Kaufmann et al., 2014) ${ }^{8}$. El cuadro 2 muestra los indicadores y su puntuación.

\section{ENTORNO, INSTRUMENTOS DE POLÍTICA Y FACTORES INSTITUCIONALES}

A pesar de la concentración de la matriz energética en solamente dos fuentes, como se observa en el Balance Energético Nacional de la Secretaría de Energía de la Nación ${ }^{9}$ (en 2014 el gas natural y el petróleo aportaron el $87 \%$ de la energía primaria interna), Argentina es uno de los países de América Latina con mayor potencial de energías renovables y donde la sustitución tiene más antigüedad. Como ya se dijo, la política energética de mediados de los años cuarenta buscaba sustituir carbón mineral y petróleo importados por fuentes más eficientes con mayor disponibilidad local. Dubrovsky (2004) señala que a finales de esa década y comienzos de la siguiente, con la finalización de la Segunda Guerra Mundial, el riesgo que la dependencia energética externa imponía a la industrialización reforzó la necesidad del autoabastecimiento. Así, las políticas de sustitución y la disponibilidad interna de recursos gasíferos e hidráulicos redujeron la participación del carbón mineral y aumentaron la del gas natural y la energía hidráulica con un impacto ambiental favorable, situación que mejoró aún más en los años noventa, cuando la generación eléctrica se orientó a tecnologías de ciclo combinado que utilizaban el abundante gas natural.

\footnotetext{
${ }^{6}$ Para más información sobre el Climatescope Report ver [http://global-climatescope. org/en/].

${ }_{7}$ Para más información ver [http://www.ren21.net/].

${ }^{8}$ Ver [http://info.worldbank.org/governance/wgi/index.aspx\#home].

${ }^{9} \operatorname{Ver}[$ http://www.energia.gov.ar/contenidos/verpagina.php?idpagina=3366].
} 
Gráfica 2

Intensidad de la emisión de co en Argentina, 1970-2012

(Gton/Mwh)

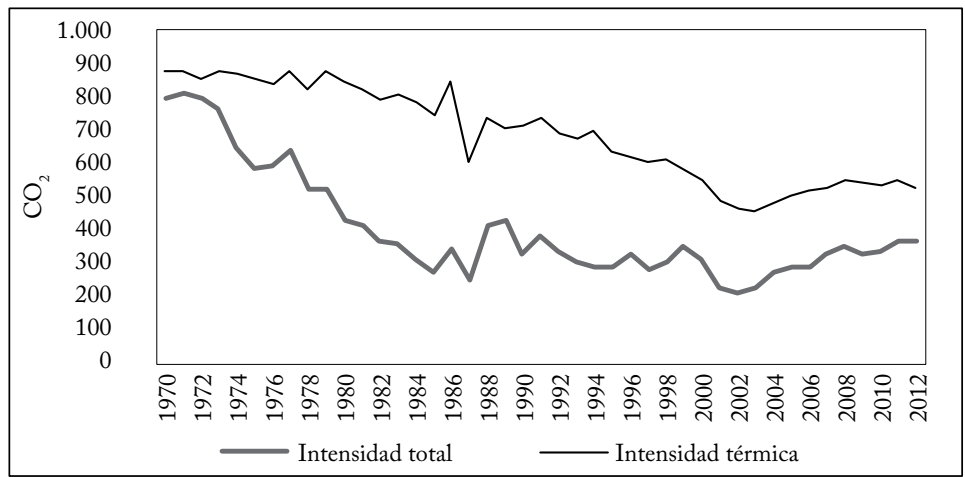

Fuente: Secretaría de la Energía ${ }^{10}$, CAmmesa $^{11}$ y Secretaría de Ambiente y Desarrollo Sustentable (2007); elaboración propia.

La gráfica 2 muestra la evolución de la intensidad de la emisión de $\mathrm{CO}_{2}$ en la generación de electricidad en Argentina entre 1970 y 2012, estimada con datos de la Secretaría de Energía de la Nación, CAMmesa e información sobre factores de emisión de la Secretaría de Ambiente y Desarrollo Sustentable (2007). Se observan dos claros momentos de reducción de dicha intensidad, en 1970-1983 y en 1990-2002, resultado de las políticas de sustitución y de la difusión de las tecnologías de ciclo combinado en la generación de electricidad. Sin embargo, la intensidad aumenta después de 2002. Esta reversión de la tendencia es un resultado del aumento de la demanda de energía en un contexto de congelación de precios de los energéticos, y del uso de combustibles líquidos para solventar la falta de gas natural (Recalde, 2011b). Esta descripción refuerza la importancia de promover las FNRE para reducir el impacto ambiental del sector eléctrico.

El cuadro 3 recopila los diferentes instrumentos de política energética utilizados para la promoción de las FNRE en el país por periodos presidenciales en las últimas tres décadas, y resume las principales condiciones del entorno en cada momento. La periodización seleccionada para el estudio se debe a que el objetivo es analizar en qué medida los cambios político-institucionales determinaron condiciones de borde diferentes para la política y cuál ha sido su impacto final.

\footnotetext{
10 [http://www.energia.gov.ar/home/].

${ }^{11}$ [http://www.cammesa.com.ar/].
} 


\section{Cuadro 3}

Condiciones de entorno e instrumentos utilizados, Argentina 1983-2015

\begin{tabular}{|c|c|c|c|c|}
\hline Periodo & Gobierno & $\begin{array}{l}\text { Principales condiciones } \\
\text { del entorno }\end{array}$ & Política/Instrumento & Análisis del instrumento \\
\hline 1983-1989 & $\begin{array}{l}\text { Constitucional } \\
\text { Finalización } \\
\text { anticipada del } \\
\text { mandato } \\
\text { Dr. Raúl Alfonsín }\end{array}$ & $\begin{array}{l}\text { Regreso al régimen } \\
\text { democrático } \\
\text { Alta inflación (1988: } \\
342 \% \text { ). } \\
\text { Altos niveles de endeu- } \\
\text { damiento (1989: } 82 \% \text { del } \\
\text { PIB). } \\
\text { Conflictos económicos y } \\
\text { sociales, y . Conflicto en } \\
\text { torno a la organización } \\
\text { del sector energético al } \\
\text { final del periodo. }\end{array}$ & $\begin{array}{l}\text { Programa Nacional de } \\
\text { Uso Racional (elabora- } \\
\text { ción pero no implementa- } \\
\text { ción). } \\
\text { Creación del Centro Re- } \\
\text { gional de Energía Eólica } \\
\text { (CREE). } \\
\text { Inventario de peque- } \\
\text { nos aprovechamientos } \\
\text { hidroeléctricos. }\end{array}$ & $\begin{array}{l}\text { Se desarrollaron princi- } \\
\text { palmente instrumentos } \\
\text { relacionados con el au- } \\
\text { mento de la información } \\
\text { disponible. }\end{array}$ \\
\hline 1989-1999 & $\begin{array}{l}\text { Constitucional } \\
\text { Reelección } \\
\text { Dr. Carlos Saúl } \\
\text { Menem }\end{array}$ & $\begin{array}{l}\text { Ley de Reforma del } \\
\text { Estado (Ley 23.696) y } \\
\text { la Ley de Emergencia } \\
\text { Económica (Ley 23.697). } \\
\text { Ley de Convertibilidad } \\
\text { del Austral: } 1 \text { austral = } 1 \\
\text { dólar estadounidense. } \\
\text { Desregulación energética, } \\
\text { desintegración vertical } \\
\text { y privatizaciones de las } \\
\text { principales empresas } \\
\text { energéticas. } \\
\text { Caída del protagonis- } \\
\text { mo del Estado en la } \\
\text { elaboración de la política } \\
\text { energética. } \\
\text { Pérdida de capacidad re- } \\
\text { gulatoria de cumplimiento } \\
\text { en los entes de regulación } \\
\text { en el sector. }\end{array}$ & $\begin{array}{l}\text { Ley 24.224: declara de } \\
\text { interés nacional la ejecu- } \\
\text { ción del carteo geológico. } \\
\text { Desarrollo del primer } \\
\text { mapa eólico nacional. } \\
\text { Ley 25.019: declara de } \\
\text { interés la generación } \\
\text { eléctrica con energía } \\
\text { eólica, establece Feed-in } \\
\text { premiums denominados } \\
\text { en pesos argentinos, } \\
\text { exenciones impositivas y } \\
\text { contratos por } 15 \text { años. } \\
\end{array}$ & $\begin{array}{l}\text { Coexisten instrumentos } \\
\text { de aumento de la infor- } \\
\text { mación energética, con } \\
\text { instrumentos económi- } \\
\text { cos para la promoción: } \\
\text { incentivos vía precios e } \\
\text { incentivos fiscales. } \\
\text { - Problema de los incenti- } \\
\text { vos denominados en mo- } \\
\text { neda local en contextos } \\
\text { de precios bajos, lo que } \\
\text { reduce el impacto sobre } \\
\text { la rentabilidad. No supera } \\
\text { la barrera de una baja } \\
\text { rentabilidad. }\end{array}$ \\
\hline 1999-2001 & $\begin{array}{l}\text { Constitucional } \\
\text { Finalización } \\
\text { anticipada del } \\
\text { mandato del } \\
\text { Dr. Fernando de } \\
\text { la Rúa }\end{array}$ & $\begin{array}{l}\text { - Caída del nivel de } \\
\text { actividad y fuerte recesión } \\
\text { económica. } \\
\text { Altos niveles de fugas de } \\
\text { capitales. } \\
\text { Política monetaria: } \\
\text { Corralito financiero. } \\
\text { - Reducciones de finan- } \\
\text { ciamiento del Banco } \\
\text { Mundial. } \\
\text { - Conflictos económicos y } \\
\text { sociales. } \\
\text { Incrementos en los nive- } \\
\text { les de desempleo (18,3\% } \\
\text { en 2001) y pobreza (38\% } \\
\text { de las personas). } \\
\text { Interrupción del financia- } \\
\text { miento externo. }\end{array}$ & \begin{tabular}{|l} 
Programa de electrifica- \\
ción rural, PERMER.
\end{tabular} & $\begin{array}{l}\text { Instrumentos de pro- } \\
\text { moción de la inversión } \\
\text { en fnre en zonas rurales. } \\
\text { Principalmente inversión } \\
\text { pública dependiente } \\
\text { del financiamiento de } \\
\text { organismos multilaterales. }\end{array}$ \\
\hline $2002-2003$ & $\begin{array}{l}\text { Transición } \\
\text { Dr. Duhalde }\end{array}$ & $\begin{array}{l}\text { Ley de emergencia públi- } \\
\text { ca. } \\
\text { Reforma del régimen } \\
\text { cambiario. } \\
\text { Medidas de relevan- } \\
\text { cia: prohibición de la } \\
\text { indexación de deudas, } \\
\text { impuestos, precios o tari- } \\
\text { fas de bienes o servicios; } \\
\text { pesificación } 1 \text { a } 1 \text { precios } \\
\text { y tarifas de servicios } \\
\text { públicos; renegociación de } \\
\text { los contratos. } \\
\text { Altos niveles de desem- } \\
\text { pleo y pobreza. } \\
\text { Se mantiene la interrup- } \\
\text { ción del financiamiento } \\
\text { externo. }\end{array}$ & \begin{tabular}{|l} 
Sin cambios significa- \\
tivos: no existen instru- \\
mentos especíicos para \\
la inversión en energías \\
renovables.
\end{tabular} & \\
\hline
\end{tabular}




\begin{tabular}{|c|c|c|c|c|}
\hline Periodo & Gobierno & $\begin{array}{l}\text { Principales condiciones } \\
\text { del entorno }\end{array}$ & Política/Instrumento & Análisis del instrumento \\
\hline 2003-2007 & $\begin{array}{l}\text { Constitucional } \\
\text { Dr. Néstor } \\
\text { Kirchner }\end{array}$ & $\begin{array}{l}\text { Crecimiento económico } \\
\text { con contención de precios } \\
\text { de los servicios. } \\
\text { Comienzan a evidenciarse } \\
\text { síntomas de crisis energé- } \\
\text { tica en } 2004 \text {. } \\
\text { Periodos de interrupción } \\
\text { programada de abasteci- } \\
\text { miento de gas natural a } \\
\text { los usuarios interrumpi- } \\
\text { bles. } \\
\text { Inicio de los cortes de } \\
\text { suministro eléctrico no } \\
\text { programado e imprevisto } \\
\text { en los periodos de pico } \\
\text { de demanda eléctrica en } \\
\text { los mayores centros de } \\
\text { consumo del país. }\end{array}$ & $\begin{array}{l}\text { Ley } 26.123 \text { Régimen para } \\
\text { el desarrollo de la tecno- } \\
\text { logía, producción, uso y } \\
\text { aplicación del hidrógeno } \\
\text { como combustible y } \\
\text { vector de energía. } \\
\text { Plan Estratégico Nacional } \\
\text { Eólico. } \\
\text { - } \text { sig eólico. } \\
\text { Ley } 26.190 \text { : cuota } 8 \%, \\
\text { esquema de Feed-in } \\
\text { premiums (sobreprecios) } \\
\text { en pesos argentinos, } \\
\text { exenciones impositivas } \\
\text { sEe n. }{ }^{\circ} \text { 1.281/2006, la } \\
\text { cual define la prioridad } \\
\text { de abastecimiento ante } \\
\text { déficits del servicio } \\
\text { público; o la Resolución } \\
\text { sEe n. } 220 / 2007 \text { que } \\
\text { habilita la realización de } \\
\text { contratos de abasteci- } \\
\text { miento entre el mem. } \\
\text { Creación de Vientos de } \\
\text { la Patagonia I (EnARsa + } \\
\text { Gobierno de la Provincia } \\
\text { del Chubut) }\end{array}$ & $\begin{array}{l}\text { - Coexisten instrumentos } \\
\text { de incremento de la } \\
\text { información energética, } \\
\text { con instrumentos econó- } \\
\text { micos para la promoción: } \\
\text { incentivos vía precios e } \\
\text { incentivos fiscales. Se } \\
\text { incorporan instrumentos } \\
\text { cuantitativos: estableci- } \\
\text { miento de cuotas. Proble- } \\
\text { ma de los incentivos que } \\
\text { se encuentran nominados } \\
\text { en moneda local en } \\
\text { contextos de precios bajos } \\
\text { y devaluación cambiaria } \\
\text { lo que reduce el impacto } \\
\text { sobre la rentabilidad. No } \\
\text { supera la barrera de la } \\
\text { baja rentabilidad. } \\
\text { Incremento de la inver- } \\
\text { sión pública para extender } \\
\text { redes de transmisión, } \\
\text { inversión para creación } \\
\text { de empresas públicas } \\
\text { destinadas al desarrollo de } \\
\text { renovables. } \\
\text { Inversión pública en desa- } \\
\text { rrollo de parques eólicos. }\end{array}$ \\
\hline $2007-2015$ & $\begin{array}{l}\text { Constitucional } \\
\text { Reelección } \\
\text { Dra. Cristina } \\
\text { Fernández de } \\
\text { Kirchner }\end{array}$ & $\begin{array}{l}\text { Altas tasas de crecimiento } \\
\text { económico en el primer } \\
\text { periodo electoral. } \\
\text { Conflictos con institu- } \\
\text { ciones de financiamiento } \\
\text { externo: club de París, } \\
\text { conflicto con holdouts, etc. } \\
\text { Serios problemas de abas- } \\
\text { tecimiento energético. } \\
\text { Periodos de interrupción } \\
\text { programada de abasteci- } \\
\text { miento de gas natural a } \\
\text { los usuarios interrumpi- } \\
\text { bles. } \\
\text { Profundización los cortes } \\
\text { de suministro eléctrico no } \\
\text { programado e imprevisto } \\
\text { en los periodos de pico } \\
\text { de demanda eléctrica en } \\
\text { los mayores centros de } \\
\text { consumo del país. } \\
\text { Expropiación del } 51 \% \text { de } \\
\text { las acciones de la empresa } \\
\text { pertenecientes a Repsol } \\
\text { YPF s.A. } \\
\text { Incrementos de las } \\
\text { importaciones energéticas } \\
\text {-saldo de la balanza } \\
\text { energética-; impacto so- } \\
\text { bre el déficit de la balanza } \\
\text { comercial e impactos } \\
\text { sobre las reservas. }\end{array}$ & $\begin{array}{l} \\
\text { Programa de licitaciones } \\
\text { de generación de energía } \\
\text { eléctrica a partir de fuen- } \\
\text { tes renovables (GENREN): } \\
\text { subastas por 1.000 Mw } \\
\text { energías renovables: } \\
\text { esquema de Feed-in tarifs } \\
\text { en dólares estadouni- } \\
\text { denses, contratos a } \\
\text { largo plazo, exenciones } \\
\text { impositivas. } \\
\text { Resolución seE } 280 / 2008 \\
\end{array}$ & $\begin{array}{l}\text { Coexisten instrumentos } \\
\text { de precio y cantidad; } \\
\text { incentivos de precios y } \\
\text { exenciones fiscales. } \\
\text { Esquemas de precios } \\
\text { denominados en dólares, } \\
\text { lo que logra superar la } \\
\text { barrera de rentabilidad } \\
\text { de las inversiones de los } \\
\text { esquemas anteriores. } \\
\text { Persiste el problema de } \\
\text { la falta de instrumentos } \\
\text { que superen la barrera del } \\
\text { financiamiento. }\end{array}$ \\
\hline
\end{tabular}

Fuente: Recalde et al. (2015), en ese trabajo se encuentran más detalles de los instrumentos utilizados en cada periodo. 
Cabe destacar dos aspectos. Primero, en las últimas décadas las condiciones del entorno se han caracterizado por grandes periodos de volatilidad de las variables macroeconómicas, (si bien ha habido algunos cambios coyunturales), por la reducción de la financiación externa, la inestabilidad política e institucional que interrumpió dos periodos presidenciales (a finales de los ochenta y de los noventa) y falta de conciencia social. En estos periodos de crisis e inestabilidad política y económica la ruptura de los contratos fue una constante que agravó la incertidumbre.

Segundo, si bien a mediados de los noventa hay intentos de incorporar instrumentos de promoción, su uso solo se profundiza a inicios del milenio. El análisis de los instrumentos seleccionados indica que así hayan variado en los últimos años para adaptarse a las nuevas condiciones, ha habido fallas en la elección y el diseño, como la creación de feed-in premiums en la Ley 26.190, cuando los precios de generación eran muy bajos, o de primas denominadas en pesos en contextos de tasa de cambio fluctuante con el peligro de devaluaciones que reduzcan su rentabilidad (Recalde et al., 2015). En los últimos años se han modificado los instrumentos, con un diseño similar al de los demás países latinoamericanos, por ejemplo, la incorporación de esquemas feed-in tarifs en contratos de largo plazo con precios fijos denominados en dólares en la licitación de fuentes de energía nuevas y renovables (GENREN) para superar la barrera de la rentabilidad (Recalde, 2016).

Cuadro 4

La inversión en energías renovables

\begin{tabular}{lcccc}
\hline \multirow{2}{*}{ Fuente } & \multicolumn{2}{c}{ Contratados GENREN } & \multicolumn{2}{c}{ En implementación (total) } \\
\cline { 2 - 5 } & $\begin{array}{c}\text { Número de } \\
\text { proyectos }\end{array}$ & $\begin{array}{c}\text { Capacidad } \\
\text { (Mw) }\end{array}$ & $\begin{array}{c}\text { Número de } \\
\text { proyectos }\end{array}$ & $\begin{array}{c}\text { Capacidad } \\
\text { instalada (Mw) }\end{array}$ \\
\hline Eólica & 17 & 754 & 8 & 166,8 \\
PAH* & 5 & 10,6 & 32 & 284,4 \\
Fotovoltaico & 6 & 20 & 5 & 28,8 \\
Biocombustibles/biomasa & 4 & 110,4 & 2 & 18,6 \\
Total & 32 & 895 & 47 & 498,6 \\
\hline
\end{tabular}

* Incluye todos los menores de 30 mw conforme a la definición de la Ley 26.190.

Fuente: REEEP/SE/FB (2009), ENARSA, Secretaría de Energía, CAMMESA y fuentes periodísticas; elaboración propia.

Como muestra el cuadro 4, la gran mayoría de las inversiones en generación eólica, fotovoltaica y con uso de biomasa se han realizado en el marco del GENREN, lanzado por el gobierno en 2010. No obstante, cinco años después solo se han hecho efectivos 130 Mw de los 754 MW eólicos (el 17\%); 7,2 MW de los 20 MW fotovoltaicos (el 36\%); 1 MW de biogás y 1 Mw de pequeños aprovechamientos hidroeléctricos 
(РАH). A finales de 2014 la capacidad instalada de energías renovables era de apenas el 1,3\%, lejos del 8\% que se debería alcanzar en 2016, y muy lejos de muchos países latinoamericanos (Recalde, 2016).

Esta baja capacidad instalada de energías renovables y el escaso avance de los proyectos del GENREN (el 15,5\% a finales de 2014) invitan a analizar los aspectos determinantes. Puesto que, como ya se mencionó, en los últimos años se han modificado los instrumentos para superar las barreras, la atención debe centrarse en las condiciones del entorno. Para ello, el análisis de los aspectos incluidos en el cuadro 3 se complementa con el de las condiciones del entorno siguiendo el método expuesto en la sección anterior.

Gráfica 3

Rodograma del entorno y la política de energías renovables Argentina, 2014

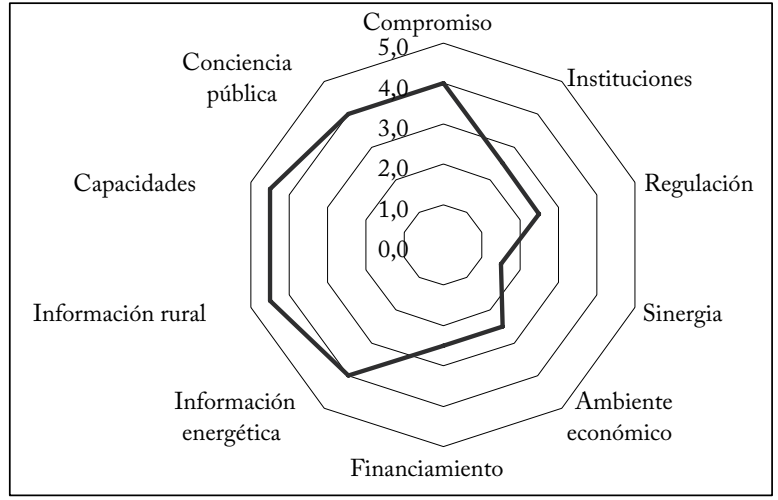

Fuente: elaboración propia.

Como muestra la gráfica 3, los puntos más débiles de las condiciones del entorno en el país corresponden a instituciones, regulación, sinergia y condiciones macroeconómicas. La falta de sinergia entre las políticas energéticas y las políticas de cambio climático hacen que el país pierda posibilidades de desarrollo y financiación para invertir en tecnologías renovables. Además, si bien en las últimas décadas Argentina ha mejorado los marcos regulatorios para incentivar la inversión en energías renovables, varios informes remarcan la baja calidad regulatoria ${ }^{12}$. Este aspecto, el contexto macroeconómico nada propicio y el escaso acceso a la financiación guardan relación directa con la debilidad institucional, en gran medida ligada a la inseguridad de los derechos de propiedad. Según BNEF et al. (2014), el riesgo de

${ }^{12}$ Ver, p. ej. el informe del Governance Index del Banco Mundial. 
mercado, la falta de alternativas de financiación, los altos subsidios, los bajos precios de los energéticos, el riesgo de incumplimiento de los contratos y la deficiente calidad de las políticas son los principales problemas que enfrenta el país.

Los aspectos más débiles de las condiciones del entorno pueden ser explicados entonces desde el punto de vista institucional. Distintos autores resaltan que la historia argentina se caracteriza por la debilidad de sus instituciones políticas y económicas, la cual ha tenido efectos negativos en el desempeño socioeconómico ${ }^{13}$.

Spiller y Tommasi (2000) muestran que el desempeño de Argentina en el siglo xx fue negativo, tanto en lo económico como en lo político e institucional. Argumentan que las instituciones generaron una estructura de gobernanza que dificulta los acuerdos intertemporales, la concreción de las decisiones de inversión y el diseño de políticas sostenibles de largo plazo. Al aumentar la inestabilidad y la inseguridad política, institucional y económica, los costos de transacción aumentan y reducen los incentivos a la inversión. London et al (2003) coinciden en que el marco institucional es deficiente, de modo que los agentes económicos contemplan altas tasas de descuento en sus decisiones intertemporales, lo que afecta el desempeño de la economía. Este deficiente marco institucional ha sido recurrente en la historia argentina. Según Blanco (2002), en más de una oportunidad la clase política ha violado los derechos de propiedad, degradado la política y la actividad legislativa y elevado la deuda pública en pos de objetivos políticos de corto plazo. Los altos niveles de inestabilidad e incertidumbre han perjudicado el desempeño industrial reciente, porque aumentan la incertidumbre y provocan una renuencia a invertir a largo plazo (Kosacoff y Ramos, 2006). Este aspecto ha sido clave en el sector energético en general y en el subsector de energías renovables en particular. La ruptura de los contratos y su incumplimiento efectivo han sido característicos en el sector energético nacional en las últimas décadas, así como las fallas de los organismos de control. Un aspecto que empezó a ser más evidente a comienzos de los noventa y se agravó en los últimos años con la ruptura de los contratos de servicios públicos y la congelación de precios de los energéticos (Recalde, 2011a; 2011b; 2011c).

El bajo nivel de la inversión en energías renovables no se debe únicamente al incipiente esquema de incentivos reformado hace poco, sino a la conjunción de condiciones del entorno, que genera un

\footnotetext{
${ }^{13}$ Ver, p. ej., Spiller y Tommasi (2000), Blanco (2002), London et al. (2003), Kosacoff y Ramos ( 2006) y London y Santos (2007).
} 
contexto poco propicio para este tipo de inversiones. Esas condiciones son, en últimas, un resultado de la baja calidad institucional del país, que ha provocado continuas rupturas de los contratos y generado incentivos negativos para la inversión. En el análisis que se sintetiza en la gráfica 1, el deficiente desempeño de las instituciones políticas y económicas ha afectado el desempeño económico y el diseño de políticas de promoción, en particular en un contexto de incertidumbre que reducen los incentivos para invertir en FNRE.

\section{REFLEXIONES FINALES}

El análisis del diseño y del desempeño de la política de energías renovables de Argentina sugiere algunos aspectos relevantes para un análisis más general y de otros países; y muestra algunas particularidades de la historia económica y política del país.

Si bien Argentina empezó a sustituir fuentes energéticas ya hace tiempo, no logró un aumento significativo de la capacidad instalada de fuentes renovables, y en los últimos años se observa incluso una reversión del impacto ambiental del sector energético por falta de una sólida política energética y ambiental.

Desde el punto de vista del diseño y la ejecución de las políticas, uno de los principales errores ha sido la selección de instrumentos que no eliminan las principales barreras a la entrada y difusión de las tecnologías. La barrera económica asociada a la baja rentabilidad de los proyectos de energías renovables no ha sido eliminada por ninguna de las políticas, salvo por el reciente plan GENREN. La introducción de feed-in premiums denominados en pesos, en un contexto de bajos precios de los energéticos y devaluaciones cambiarias, diluyó el impacto del incentivo a la inversión. El GENREN, el programa de política mejor diseñado en los últimos años, después adoptó un esquema feed-in tariff, un esquema de precios fijos denominados en dólares, que buscaba superar la barrera de rentabilidad que no eliminaron las leyes 25.019 y 26.190. Pero el programa no ha logrado los resultados deseados y el cumplimiento de sus metas es muy bajo, debido principalmente a la falta de financiación.

Gran parte del deficiente desempeño de estas políticas se debe a las condiciones del entorno, una consecuencia indirecta del marco institucional. Los principales aspectos del entorno que dificultan la expansión de las energías renovables en el país se pueden clasificar en dos categorías. Por un lado, los aspectos del entorno específicos del sector energético-ambiental: la falta de compromiso del gobierno con las energías renovables, el bajo nivel de desarrollo de las instituciones 
de energías renovables, la inexistencia de entidades especializadas en su promoción, la escasa integración entre políticas ambientales y energéticas, y la mala percepción de la capacidad de las instituciones para formular e implementar políticas de energía limpia. Por otro lado, los aspectos que conciernen a la percepción que tienen los inversionistas privados de las condiciones macroeconómicas: variaciones del índice de precios al consumidor, tasa de inflación anual, protección de los derechos de propiedad y falta de credibilidad.

Todos esos aspectos son resultado de la baja calidad de las instituciones. La inestabilidad institucional ha perjudicado el desempeño del sector energético en general, y en particular el del subsector de energías renovables. La falta de credibilidad en el sistema político, la falta de estructuras que den estabilidad al sistema económico, la imperfección de los derechos de propiedad, el incumplimiento de los contratos y la debilidad de los mecanismos de control para asegurar su cumplimiento generan expectativas negativas y reducen los incentivos para invertir en energías renovables.

El reconocimiento del efecto de las expectativas y de la confianza en las instituciones sobre la inversión hace necesarias políticas de Estado que remedien esa situación. Si no se modifican los factores mencionados, las políticas e instrumentos de promoción no darán los resultados esperados. En un contexto internacional que exige cada vez mayores inversiones en energías renovables es esencial reconocer la importancia de estos factores para mejorar las perspectivas energéticas y ambientales del país.

\section{REFERENCIAS BIBLIOGRÁFICAS}

1. Acemoglu, D. y J. Robinson. "The role of institutions in growth and development", working paper 10, International Bank for Reconstruction and Development-World Bank, 2008.

2. Altomonte, H. "Estado actual y desafíos para la promoción de la energía sostenible en América Latina y el Caribe", presentado en el foro "Energía sostenible para un desarrollo sostenible", Ciudad de Guatemala, marzo de 2007.

3. Arestis, P. "Post-Keynesian economics: Towards coherence", Journal of Economics 20, 1, 1996, pp. 111-135.

4. Barrera, M. A. "La diversificación de la matriz energética, un debate pendiente", Voces en el Fénix 2, 10, 2011, pp. 17-21.

5. Blanco, L. A. "Caracterización de la crisis argentina", Revista de Economía Institucional 4, 7, 2002, pp. 209-214

6. вNEF. "Multilateral Investment Fund part of the Inter-American Development Bank", Climatescope 2014 Report, 2014. 
7. Boldt, J. et al. Overcoming barriers to the transfer and diffusion of climate technologies, Roskilde, Unep Risø Centre on Energy, Climate and Sustainable Development, 2012.

8. Bouille, D. "Lineamientos para la regulación del uso eficiente de la energía en Argentina", Santiago de Chile, Cepal-Comisión Europea, 1999.

9. Carabelli, A. On Keynes's method, Londres, Palgrave Macmillan, 1988.

10. cepal/olade/gtz. Energía y desarrollo sustentable en América Latina y el Caribe: enfoques para la politica energética, Santiago de Chile, 1997.

11. Cherni, J. A. "Promotion of renewable energy in Latin America: Policy and lessons", J. Haselip et al., eds., Diffusion of renewable energy technologies. Case studies of enabling frameworks in developing countries, Roskilde, unep Risø Centre on Energy, Climate and Sustainable Development, 2011, pp. 113-128.

12. Corrigan, G. et al. "Assessing progress toward sustainable competitiveness”, K. Schwab, ed., The global competitiveness report 2014-2015, Ginebra, wef, 2014.

13. Crawford, S. y E. Ostrom. "A grammar of institutions", American Political Science Review 89, 3, 1995, pp. 582-600.

14. Davidson, P. "A technical definition of uncertainty and the long-run non neutrality of money", Cambridge Journal of Economics 12, 3, 1988, pp. 329-337.

15. Davidson, P. “Is probability theory relevant for uncertainty?”, Journal of Economic Perspectives 5, 1, 1991, pp. 129-143.

16. Del Río, P. y P. Linares. "Back to the future? Rethinking auctions for renewable electricity support”, Renewable and Sustainable Energy Reviews 35, 2014, pp. 42-56.

17. Del Río, P. y P. Mir A. "Combinations of support instruments for renewable electricity in Europe: A review", Renewable and Sustainable Energy Reviews 40, 2014, pp. 287-295.

18. DCLG. "Multi-criteria analysis: A manual", Londres, Department for Communities and Local Government, 2009.

19. Dixit, A. y R. Pindyck. Investment under uncertainty, Princeton, NJ, Princeton University Press, 1994.

20. DOI/IEA. "Energy-related carbon dioxide emissions", International Energy Outlook 2013, cap. 9, Washington DC, IEA, 2013.

21. Dubrovsky, H. "The role of renewable energy in electricity access for the poor in Argentina”, primer borrador, preparado para la conferencia Acceso a la Energía de la wgnesd, Bonn, 2004.

22. Duguma, L. A. et al. "A systematic analysis of enabling condition for synergy between climate change mitigation and adaptation measures in developing countries”, Environmental Science EF Policy 42, 2014, pp. 138-148.

23. Fouquet, D. "Policy instruments for renewable energy - From a European perspective”, Renewable Energy 49, C, 2013, pp. 15-18

24. Foxon, T. et al. "Energy technology innovation: A systems perspective - Report for the Garnaut Climate Change Review", Garnaut Review, diciembre de 2007. 
25. IEE-usp, FB. Estudio de la oferta y demanda de energía. Energía: Una visión sobre los retos y oportunidades en América Latina y El Caribe, CAF, 2013, [http://repositorio.cepal.org/bitstream/handle/11362/1505/1/ Energia_CAF_CEPAL.pdf].

26. James, C. S. "Estado de la industria argentina de energías renovables", The Clean Energy Report, Buenos Aires, 2011.

27. Jacobs, D. M. et al. "Analysis of renewable energy incentives in the Latin America and Caribbean region: The feed-in tariff case", Energy Policy, 60, 2013, pp. 601-610.

28. Kaufmann, D.; A. Kraay y M. Mastruzzi. The Worldwide Governance Indicators (wgi), World Bank, 2014, [http://info.worldbank.org/governance/wgi/index.aspx\#home].

29. Kosacoff, B. y A. Ramos. "Comportamientos microeconómicos en entornos de alta incertidumbre: la industria argentina”, documento de proyecto, Santiago de Chile, Cepal, 2006.

30. London, S.; F. Poinsot y L. Straguzzi. "Instituciones y desempeño económico: algunas reflexiones para el caso argentino", Anales, Asociación Argentina de Economía Política, Mendoza, 2003.

31. London, S. y M. M. Santos. "Desarrollo e instituciones precarias: la Argentina de los 90", Economía y Sociedad 12, 20, 2007, pp. 129-158.

32. London, S. y M. Rojas. "Un nuevo desafío a la gobernabilidad: instituciones, gobernanza y desarrollo”, presentado en el Congreso de la Asociación de Economía para el Desarrollo de Argentina, Buenos Aires, mayo de 2015.

33. Mohn, K. y B. Misund. "Investment and uncertainty in the international oil and gas industry", Energy Economics 31, 2, 2009, pp. 240-248.

34. North, D. y R. Thomas. El nacimiento del mundo occidental, Madrid, Siglo XXI, 1980.

35. olade/cepal/gtz. Energía y desarrollo sustentable en ALyC: Guía para la formulación de politicas energéticas, Santiago de Chile, Cepal, 2003.

36. Ostrom, E. "Investing in capital, institutions, and incentives", C. Clague, ed., Institutions and economic development: Implications of a new-institutional economics approach for growth, poverty reduction, democracy, and external assistance, College Park, MD, University of Maryland, 1996.

37. Ostrom, E. "Institutional analysis and development: Elements of the framework in historical perspective", C. Crothers, ed., Historical developments and theoretical approaches in sociology/social theory, Auckland, University of Technology, 2010.

38. Parigi, G.; M. E. Bontempi y T. Golinelli. "Why demand uncertainty curbs investment: Evidence from a panel of Italian manufacturing firms" [2007], Journal of Macroeconomics 32, 1, 2010, pp. 218-238.

39. Painuly, J. P. "Barriers to renewable energy penetration; a framework for analysis", Renewable Energy 24, 1, 2001, pp. 73-89.

40. Painuly, J. P. y J. V. Fenhann. Implementation of Renewable Energy Technologies. Opportunities and Barriers, Roskilde, Unep Risø Centre on Energy, Climate and Sustainable Development, 2002. 
41. Polski, M. M. y E. Ostrom. "An institutional framework for policy analysis and design", Workshop in political theory and policy analysis, Indiana University, Bloomington, 1998.

42. Reeep/se/fB. República Argentina. Energías renovables: diagnóstico, barreras y propuestas, Buenos Aires, Secretaría de Energía, 2009.

43. Recalde, M. Y. "Determinantes de la inversión en exploración de hidrocarburos: un análisis del caso argentino", Cuadernos de Economía 34, 94, 2011a, pp. 40-52.

44. Recalde, M. Y. "Energy policy and energy market performance: The Argentinean case”, Energy Policy 39, 6, 2011b, pp. 3860-3868.

45. Recalde, M. Y. Sistemas energéticos, mercado y Estado: el rol de los recursos naturales energéticos y la politica energética en el caso argentino, Berlín, Editorial Académica Española, 2011c.

46. Recalde, M. Y. y J. Ramos-M. "Going beyond energy intensity to understand the energy metabolism of nations: The case of Argentina", Energy 37, 1, 2012, pp. 122-132.

47. Recalde, M. Y.; D. Bouille y L. Girardín. "Limitaciones para el desarrollo de energías renovables en Argentina", Problemas del Desarrollo 46, 183, 2015, pp. 89-115.

48. Recalde, M. Y. "The different paths for renewable energies in Latin American countries: The role of the enabling environment and the design of promoting instruments", wires Energy and Environment 5, 2016, pp. 305-326.

49. Reister, D. "The link between energy and GDP in developing countries”, Energy 12, 6, 1987, pp. 427-433.

50. Ren21. Renewables 2015. Global Status Report, París, REN21 Secretariat, 2015.

51. Rockström, J. et al. "Planetary boundaries: Exploring the safe operating space for humanity", Ecology and Society 14, 2, 2009, art. 32.

52. Salgado, R. y H. Altomonte. Indicadores de sustentabilidad 1990-1999, Santiago de Chile, Cepal, 2001,

53. Secretaría de Ambiente y Desarrollo Sustentable. Segunda comunicación nacional de la República Argentina a la Convención Marco de las Naciones Unidas sobre Cambio Climático, Buenos Aires, 2007.

54. Spiller, P. y M. Tommasi. "El funcionamiento de las instituciones políticas y las políticas públicas en la Argentina: una aproximación desde la nueva economía institucional”, Desarrollo Económico 40, 159, 2000, pp. 425-464.

55. Tahvonen, O. y S. Salo. "Economic growth and transitions between renewable and nonrenewable energy resources", European Economic Review 45, 8, 2001, pp. 1379-1398.

56. UnEP. A practical framework for planning pro-development climate policy, Copenhague, United Nations Environment Programme, 2011.

57. Van Dril, T. y X. van Tilburg. "Renewable energy: Investing in energy and resource efficiency", Towards a green economy, United Nations Environment Programme, Copenhague, 2011, pp. 201-237.

58. Vatn, A. "Combining post Keynesian, ecological and institutional economics perspectives", R. P. Holt et al., eds., Post Keynesian and 
ecological economics confronting environmental issues, Cheltenham, Edward Elgar, 2009, pp. 114-140.

59. Yifu L., J. y F. Nugent. "Institutions and economic development", Handbook of Development Economics 3, parte A, 1995, pp. 2301-2370.

60. Zaballa R., M. et al. Enabling frameworks and barrier analysis for low carbon development strategies (lcds) and nationally appropriate mitigation actions (namas), Roskilde, UneP Risø Centre on Energy, Climate and Sustainable Development, 2014. 